Revista Energia na Agricultura

ISSN 1808-8759

\title{
CONSTRUÇÃO E CALIBRAÇÃO DE UM INCLINÔMETRO PARA USO EM TRATORES AGRÍCOLAS COMO INSTRUMENTO DE SEGURANÇA ${ }^{1}$ FABRÍCIO LEITE ${ }^{2}$; JOÃO E. G. DOS SANTOS ${ }^{3} \&$ KLÉBER P. LANÇAS $^{4}$
}

RESUMO: Os tratores como fonte de potência, para o tracionamento e/ou acionamento de máquinas e implementos agrícolas dão origem a conjuntos motomecanizados, os quais têm sido utilizados de forma crescente na agricultura brasileira. Apesar do grande avanço tecnológico, o aumento do número de tratores agrícolas trouxe aspectos positivos e alguns negativos, entre estes últimos, destaca-se o surgimento de uma nova fonte de acidentes de trabalho, cuja importância é dada pelos danos físicos que causam ao operador. Portanto, o objetivo deste trabalho foi projetar, desenvolver e calibrar um inclinômetro, o qual indicava a inclinação do terreno, como sensor de segurança para tratores agrícolas com o intuito de evitar tombamentos laterais, para a preservação do operador e da máquina. O inclinômetro construído e avaliado mostrou-se eficiente para indicação da inclinação do terreno em operações de campo com tratores agrícolas, com pequena oscilação do ângulo e tempo de resposta suficiente para a tomada de decisão do operador em situação de iminência de tombamento lateral da máquina.

Palavras-chave: Tombamento lateral, máquinas, segurança agrícola.

\footnotetext{
${ }^{1}$ Parte da tese de doutorado do primeiro autor intitulada: Construção de um inclinômetro para avaliar o efeito da declividade lateral no desempenho de tratores agrícolas

${ }^{2}$ Aluno do Programa de Pós-Graduação em Agronomia - Energia na Agricultura - FCA/UNESP, Botucatu/SP, Brasil, - fleite2@uem.br

${ }^{3}$ Orientador e docente do Departamento de Engenharia - FEB/UNESP, Bauru/SP - Brasil, guarneti@,feb.unesp.br

${ }^{4}$ Co-orientador e docente do Departamento de Engenharia Rural - FCA/UNESP, Botucatu/SP - Brasil, kplancas@,fca.unesp.br
} 


\section{CONSTRUCTION AND CALIBRATION OF AN INCLINOMETER TO USE IN AGRICULTUR-} AL TRACTORS AS SAFETY INSTRUMENT

SUMMARY: The tractors are the main potency source, for the traction of tillage equipments and their use are increasing more and more in Brazilian agriculture. In spite of the great technological progress the increase of the number of agricultural tractors brought positive aspects and some negative, among these last ones it stands out the appearance of a new source of work accidents, which importance is given by the physical damages caused to the operator. Therefore the objective of this work was to project and develop an inclinometer, which indicates the inclination of the land, as safety sensor for agricultural tractors to avoid lateral hollovers, through a resonant sign, for the operator and the machine preservation. The built and evaluated inclinometer showed to be efficient for inclination indication of different areas during field operations with agricultural tractors, small angle oscillation and enough answer time to help operators to take decisions in imminence situation of lateral machine hollovers.

Keywords: Lateral hollovers, machine, safety agricultural.

\section{INTRODUÇÃO}

As causas dos acidentes com conjuntos tratorizados são definidas como sendo as condições ou atitudes inseguras que, se corrigidas a tempo, evitariam o acidente (SCHLOSSER e DEBIASI, 2001). O ato inseguro, conforme Zócchio (1971) e Cutuli et al. (1977), é a maneira como as pessoas se expõem, consciente ou inconscientemente, a acidentes. Condições inseguras, segundo os mesmos autores, são as características do meio onde o trabalho é executado que comprometem a segurança do trabalhador. Vários estudos têm indicado que, aproximadamente, 15 e $85 \%$ dos acidentes, respectivamente, são causados por condições e atos inseguros, independentemente do setor produtivo considerado (CUTULI et al.,1977; MÁRQUEZ, 1990; SCHLOSSER e DEBIASI, 2001; SANTOS, 2004).

Segundo Hunter (1992), o trator, que ocupa um lugar de destaque no maquinário agrícola, pelo fato de operar em terrenos muitas vezes acidentados, faz com que os limites de segurança sejam facilmente atingidos. 
De acordo com Mialhe (1980), o estudo das forças em equilíbrio que agem sobre o chassi do trator ajuda na compreensão do desempenho dessas máquinas em sua utilização no campo.

Conforme Corrêa e Ramos (2003), os acidentes relatados em um levantamento realizado no Estado de São Paulo, no ano de 2001, estariam geralmente associados ao uso de máquinas agrícolas, estando o trator envolvido em mais da metade dos casos (60,5\%), sendo expressivo o índice de tombamentos de tratores agrícolas $(28,93 \%$ em relação ao total de casos de acidentes e $47,83 \%$ em relação ao total de casos com tratores).

Conforme Gaiotto (2003), a capacidade de vencer fortes inclinações laterais é uma das características mais importantes em um utilitário "fora de estrada". A abordagem errada de um trecho muito inclinado poderá causar o tombamento do veículo. Isto porque a maioria dos veículos "fora de estrada" possuem o Centro de Gravidade, ou Baricentro, mais alto que os carros de passeio. Portanto, o mesmo autor comenta que, pode-se instalar um acessório conhecido como inclinômetro, que mostra as inclinações do terreno em aclives, declives e inclinações laterais e que, existem modelos avulsos vendidos no mercado especializado, e em alguns veículos o equipamento vem montado no painel, como em algumas pick-ups.

Segundo Pinho (2005), os inclinômetros medem a inclinação de um objeto a partir de uma posição anterior onde o pêndulo estava parado. Há várias maneiras de implementar um medidor de inclinação. A mais simples delas é construir "pêndulos" utilizando potenciômetros. Estes medidores de inclinação podem, por exemplo, ser utilizados em veículos para medir aceleração e alterações de direção durante a trajetória. Dependendo da aplicação, estas informações podem ser usadas para a determinação de posição. Outra forma de implementação destes inclinômetros é a utilização de sensores, semelhantes a bússolas, capazes de captar ação do campo magnético da terra.

Ot e Lladó (2001) desenvolveram um inclinômetro de baixo custo e com uma resolução entre $1^{\circ}$ ou $2^{\circ}$ sobre um intervalo entre $\left(-45^{\circ},+45^{\circ}\right)$ utilizando extensômetros, sendo que o princípio de funcionamento de um extensômetro baseia-se na variação da resistência de um condutor ou semicondutor quando se submete a um esforço mecânico.

Celeste (2002) implementou um inclinômetro microprocessado, construído a partir de sensores de aceleração com o objetivo de testar acelerômetros fabricados em chips em sistemas de navegação inercial como, por exemplo, o que permite a navegação de um robô submarino. O mesmo autor concluiu que é totalmente viável a implementação, por apresentar baixo custo, porque pode ser montado em uma simples placa de circuito impresso de pequena dimensão.

Portanto, o objetivo deste trabalho foi construir e calibrar um sensor que indique a inclinação lateral do terreno a ser explorado, evitando assim possíveis tombamentos laterais de tratores agrícolas, preservando desta forma, o operador e a máquina. 


\section{MATERIAL E MÉTODOS}

Este trabalho foi desenvolvido no Núcleo de Ensaios de Máquinas e Pneus Agroflorestais (NEMPA) pertencente à Faculdade de Ciências Agronômicas Universidade Estadual Paulista, Campus de Botucatu, com o objetivo de desenvolver um inclinômetro utilizando-se, potenciômetro de precisão munido de um pêndulo na sua extremidade, sendo estes enclausurados em uma caixa de acrílico com transferidor para avaliar a declividade do trator em relação ao terreno no sentido transversal (declividade lateral à direita). O transferidor foi desenvolvido através de programa computacional para desenhos (AutoCAD 2002), onde os ângulos estão representados numericamente e por escala de cores indicativas de trafegabilidade em terrenos inclinados. A faixa verde indicava posição de tráfego normal sem iminência de tombamento, faixa amarela atenção e faixa roxa indicava perigo, ou seja, o trator está em iminência de tombamento. A Figura 1 ilustra o transferidor desenvolvido.

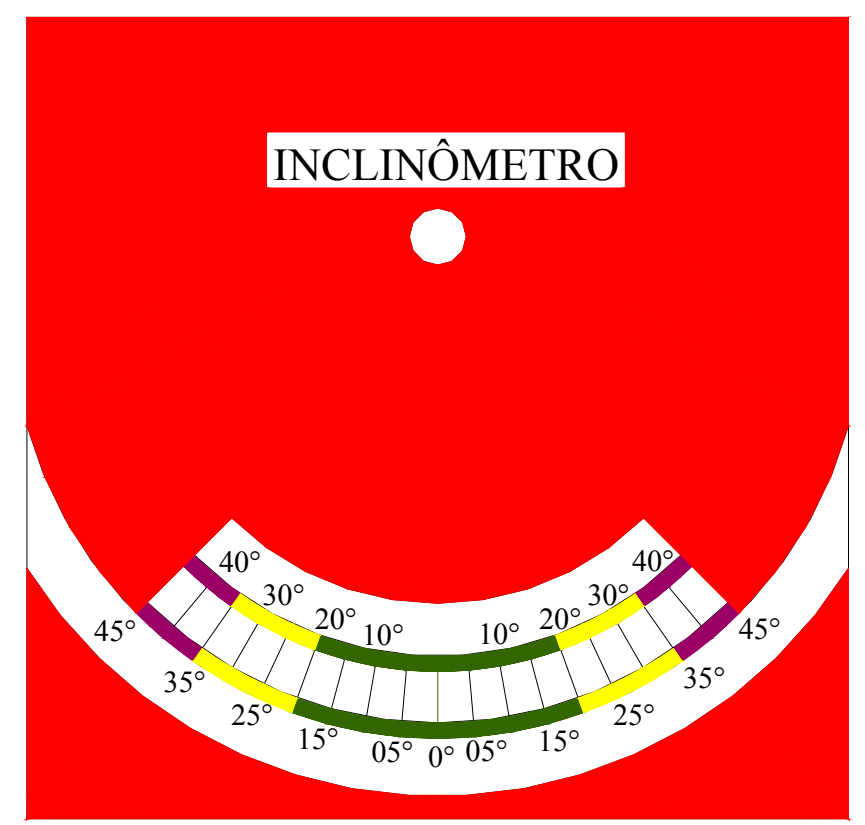

Figura 1 - Transferidor desenvolvido para o inclinômetro.

O projeto do inclinômetro, constituiu na utilização de uma massa de chumbo com $20 \mathrm{~g}$ e uma haste de ferro chato, ABNT 1020 de 25,4 mm e espessura de $1 \mathrm{~mm}$, para a fixação do chumbo e formar o pêndulo. O comprimento do pêndulo foi calculado pela Equação 33, conforme Silva (2006). 
$\mathrm{L}=\left(1 / 4 \cdot \pi^{2}\right) \cdot\left(\mathrm{g} / \mathrm{f}^{2}\right)$

Onde:

$\mathrm{L}=$ comprimento do pêndulo $(\mathrm{m})$

$\mathrm{g}=$ aceleração da gravidade $\left(\mathrm{m} \cdot \mathrm{s}^{-2}\right)$

$\mathrm{f}=$ freqüência de aquisição do sistema de aquisição de dados (s)

O inclinômetro foi calibrado em confronto com uma rampa de madeira com $1030 \mathrm{~mm}$ de comprimento, a qual permitia uma regulagem de altura através de uma rosca sem fim com borboleta de fixação, formando desta maneira um triângulo retângulo. Desta forma, a rampa permitia formar várias inclinações, tendo como valor fixo a hipotenusa (compimento da rampa de $1030 \mathrm{~mm}$ ) e variando a altura da rampa, cateto oposto, obtendo-se assim um triângulo retângulo. Sobre esta rampa de madeira, foi fixado o inclinômetro para obter os dados para a calibração do aparelho. Para a determinação da estabilidade do inclinômetro, colocou-se um trator de $89 \mathrm{~kW}$ de potência nominal do motor em movimento, sob uma inclinação lateral de 25 graus com o aparelho instalado sobre o painel. Posteriormente, com o trator parado e em marcha lenta obtiveram os dados de inclinação durante cinco minutos. O tempo de resposta foi determinado com o trator deslocando, com o inclinômetro instalado sobre o painel de instrumentos do trator, em uma inclinação lateral de 20 graus. Posteriormente deslocando-se o trator em uma inclinação de 15 graus onde através dos dados foi verificado qual foi o tempo de resposta do aparelho para mudanças de inclinação. Esses sinais foram enviados a um sistema eletrônico de aquisição de dados "CR23X - microlloger" da CAMPBELL SCIENTIFIC, com 4 canais contadores de pulso e entrada de 12 canais analógicos diferenciais e 24 não diferenciais, onde foram armazenados os dados de inclinação e tempo.

\section{RESULTADOS E DISCUSSÃO}

Neste capítulo, estão apresentados os desenhos detalhados do inclinômetro projetado, bem como os resultados da calibração do inclinômetro para uso em tratores agrícolas. A Figura 2 apresenta o esquema de montagem do inclinômetro na caixa de acrílico, com vistas frontais e laterais, respectivamente.

As dimensões da caixa de acrílico, onde foi alojado o inclinômetro, eram 120 × 120 x $120 \mathrm{~mm}$. Nas Figuras 3, 4 e 5 estão apresentados os desenhos de dimensionamento de todo o aparelho. 

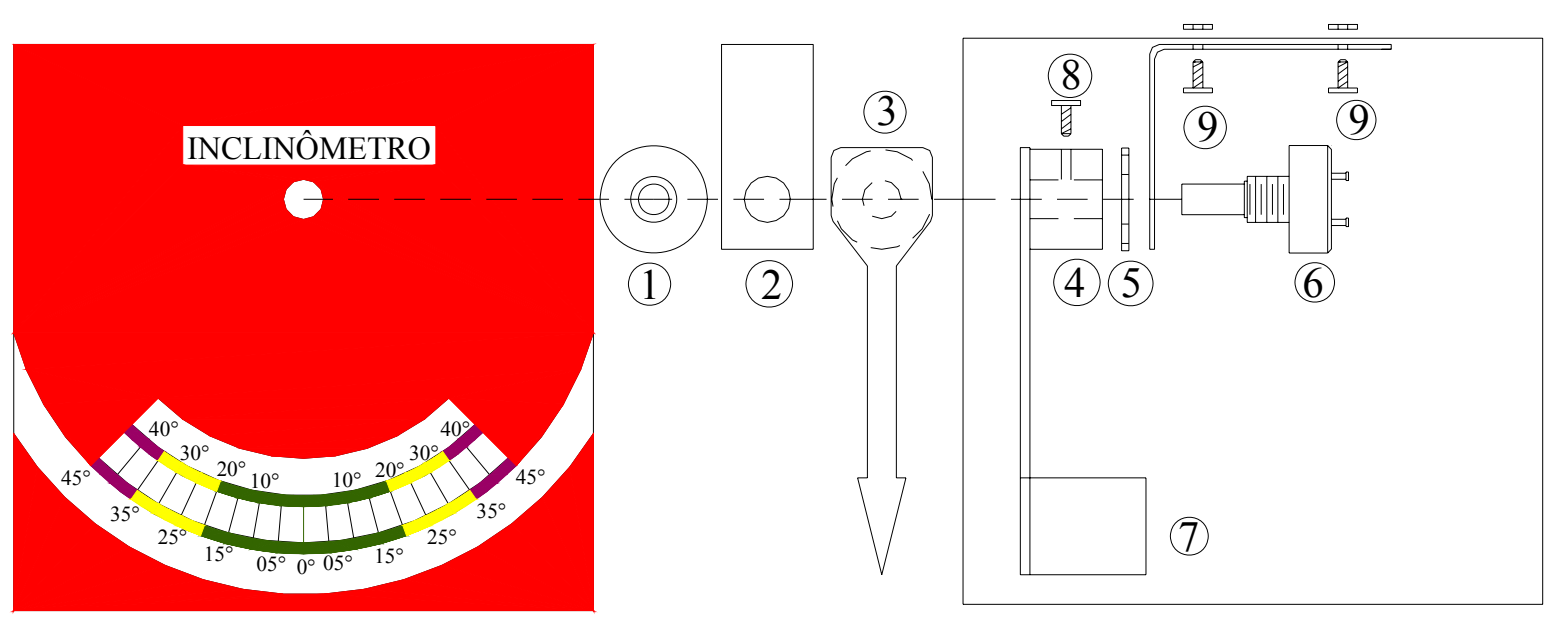

Figura 2 - (1) Vista frontal do potenciômetro, (2) Vista frontal da chapa de fixação do aparelho na caixa de acrílico, (3) Vista frontal do pêndulo, (4) Vista lateral da bucha de fixação do potenciômetro, (5) Arruela de fixação do potenciômetro, (6) Vista lateral do potenciômetro, (7) Massa de chumbo, (8) e (9) Parafusos de fixação.
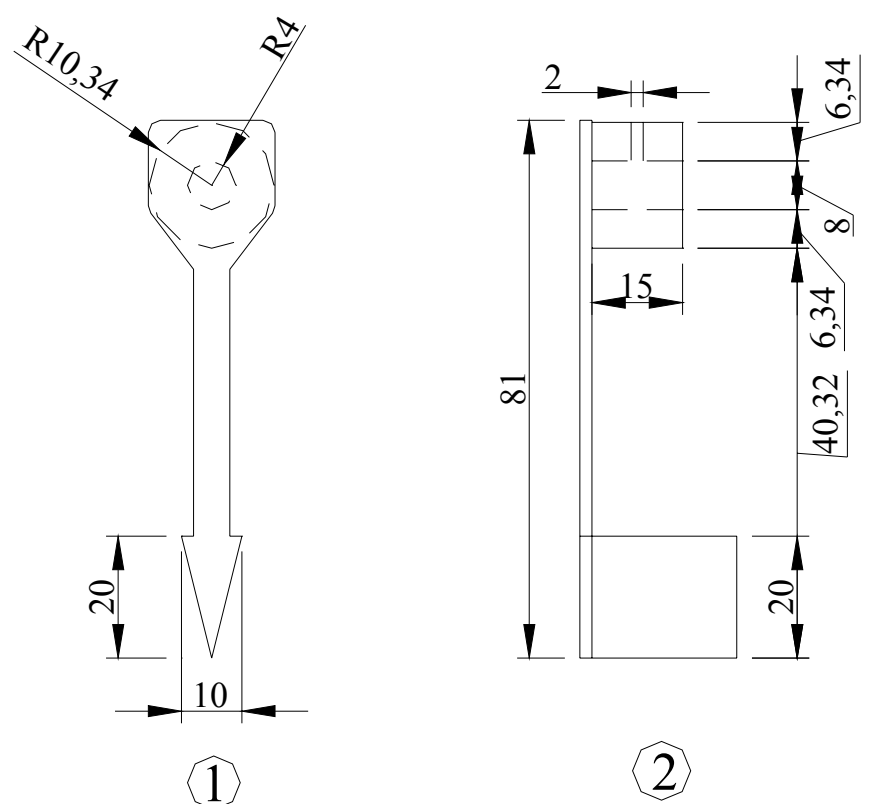

Figura 3 - (1) Vista frontal e (2) Vista lateral do pêndulo utilizado no inclinômetro (cotas em milímetros). 


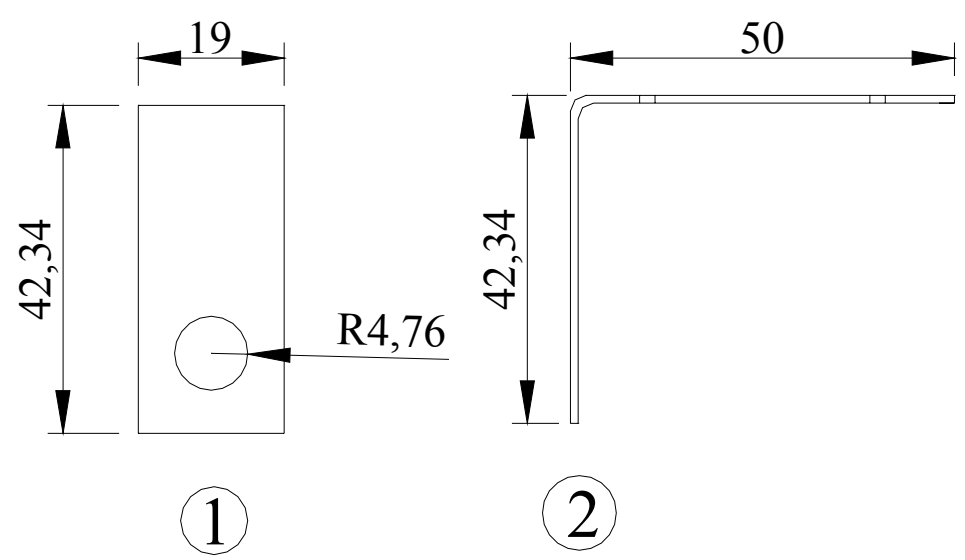

Figura 4 - (1) Vista frontal e (2) Vista lateral da chapa de fixação do aparelho na caixa de acrílico (cotas em milímetros).

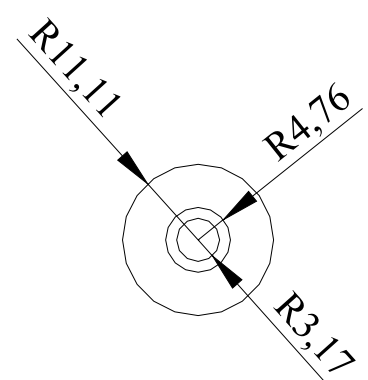

1

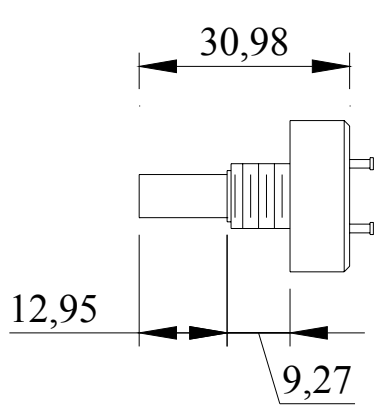

2

Figura 5 - (1) Vista frontal e (2) Vista lateral do potenciômetro utilizado no inclinômetro (cotas em milímetros).

O resultado encontrado da calibração do inclinômetro quando comparado à rampa de madeira, graduada com transferidor e onde foi fixado o inclinômetro, gerou um valor de 0,897 graus para o multiplicador (multiply) e $+1,257$ graus para o offset, os quais foram utilizados no sistema de aquisição de dados, conforme observado na Figura 6. 


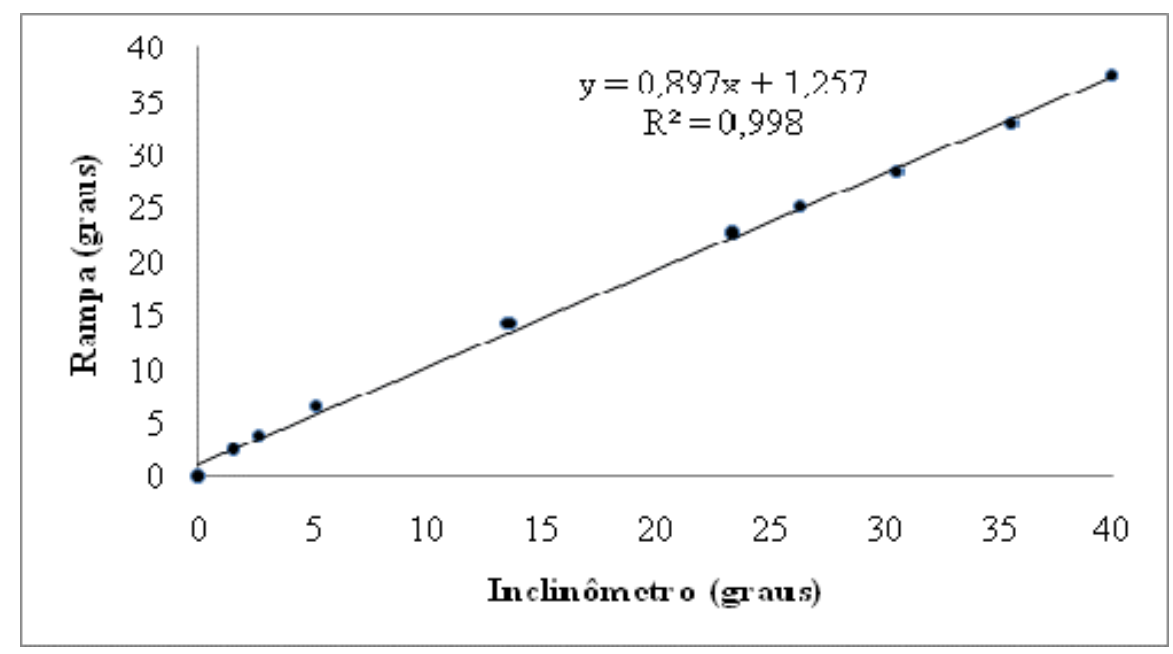

Figura 6 - Curva de calibração do inclinômetro.

A faixa de leitura do inclinômetro para declives laterais foi de $-45^{0}$ a $+45^{0}$ de inclinação, apresentando um erro de $0,1 \%$, com resolução analógica (transferidor na caixa de acrílico) de $5^{0}$ e resolução digital (sistema de aquisição de dados - microlloger) de $0,001^{0}$. Um inclinômetro capacitivo de fabricação micro-mecânica, desenvolvido por Benz et al. (2005), apresentou um erro de $0,2 \%$ para uma faixa de leitura de $-45^{0}$ a $+45^{0}$ de inclinação.

O inclinômetro projetado e desenvolvido pode ser melhor visualizado na Figura 7, que mostra como o aparelho foi instalado e fixado sobre o painel de instrumentos do trator ensaiado.

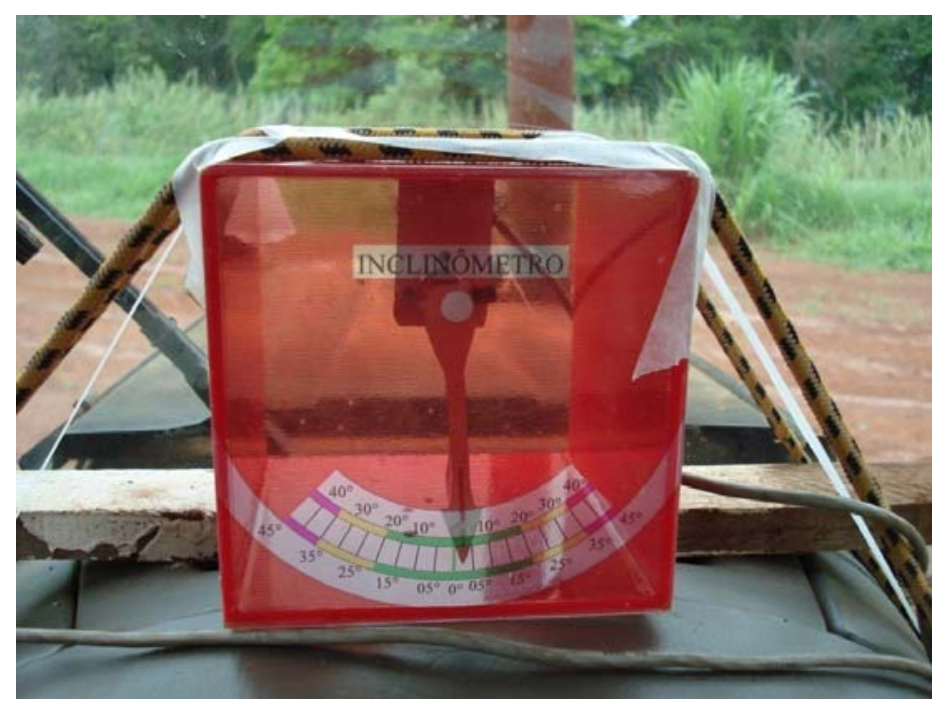

Figura 7 - Inclinômetro desenvolvido para determinação da inclinação lateral do terreno. 
O tempo de resposta que o inclinômetro apresentou na mudança da inclinação de 20,402 graus para a inclinação de 15,544 graus foi de $0,275 \mathrm{~s}$, ou seja, $275 \mathrm{~ms}$ conforme pode ser visualizado na Figura 8. Entretanto, Billat et al. (2002) desenvolveram um inclinômetro térmico de fluxo de transporte livre para fluídos, em que o tempo de resposta do sensor foi de $600 \mathrm{~ms}$.

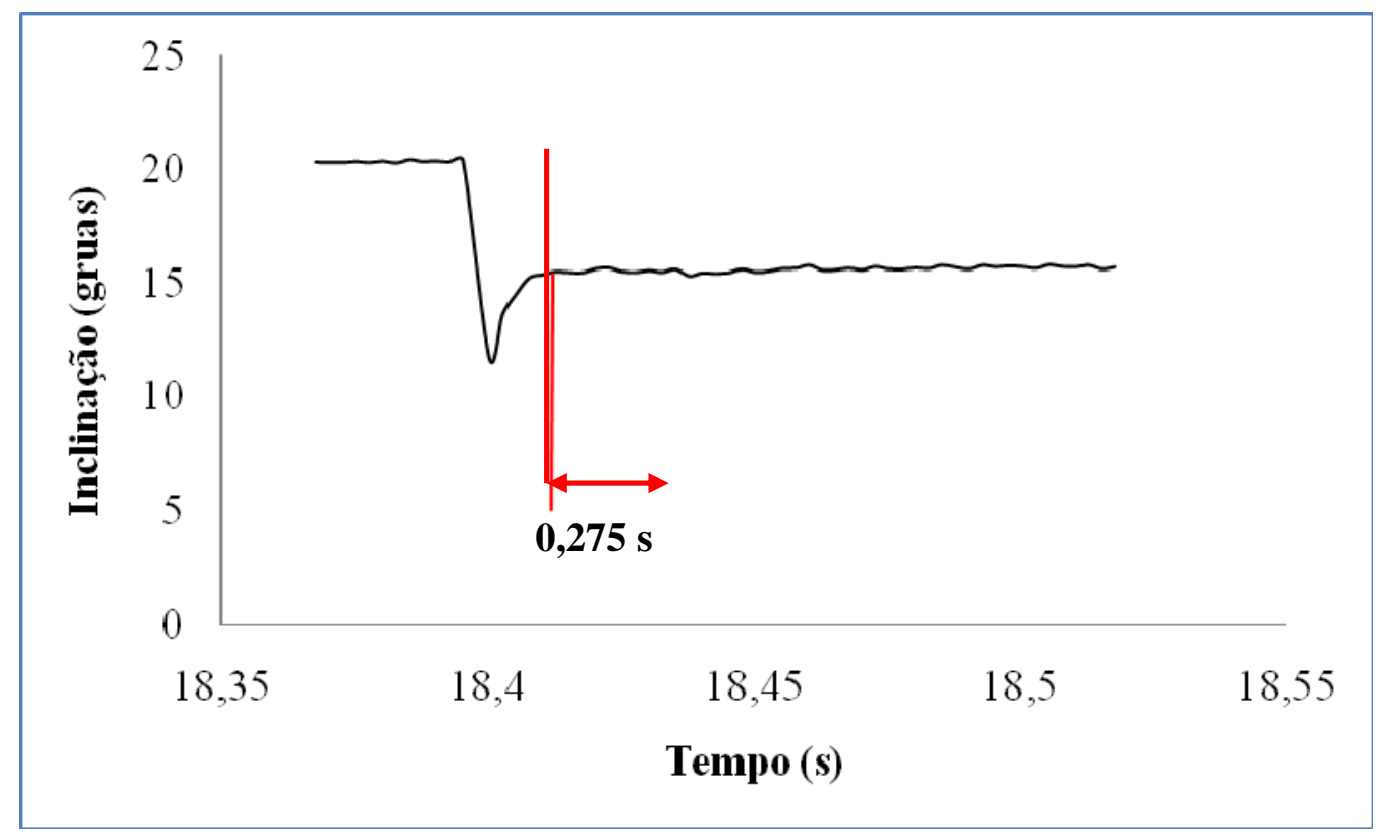

Figura 8 - Tempo de resposta apresentado pelo inclinômetro.

O inclinômetro apresentou-se com uma oscilação máxima de ângulo de 1,429 graus em um intervalo de 5 minutos de teste, conforme pode ser visualizado na Figura 9. Esta oscilação pode ser devido à vibração do motor do trator agrícola, principalmente por estar o trator em marcha lenta. 


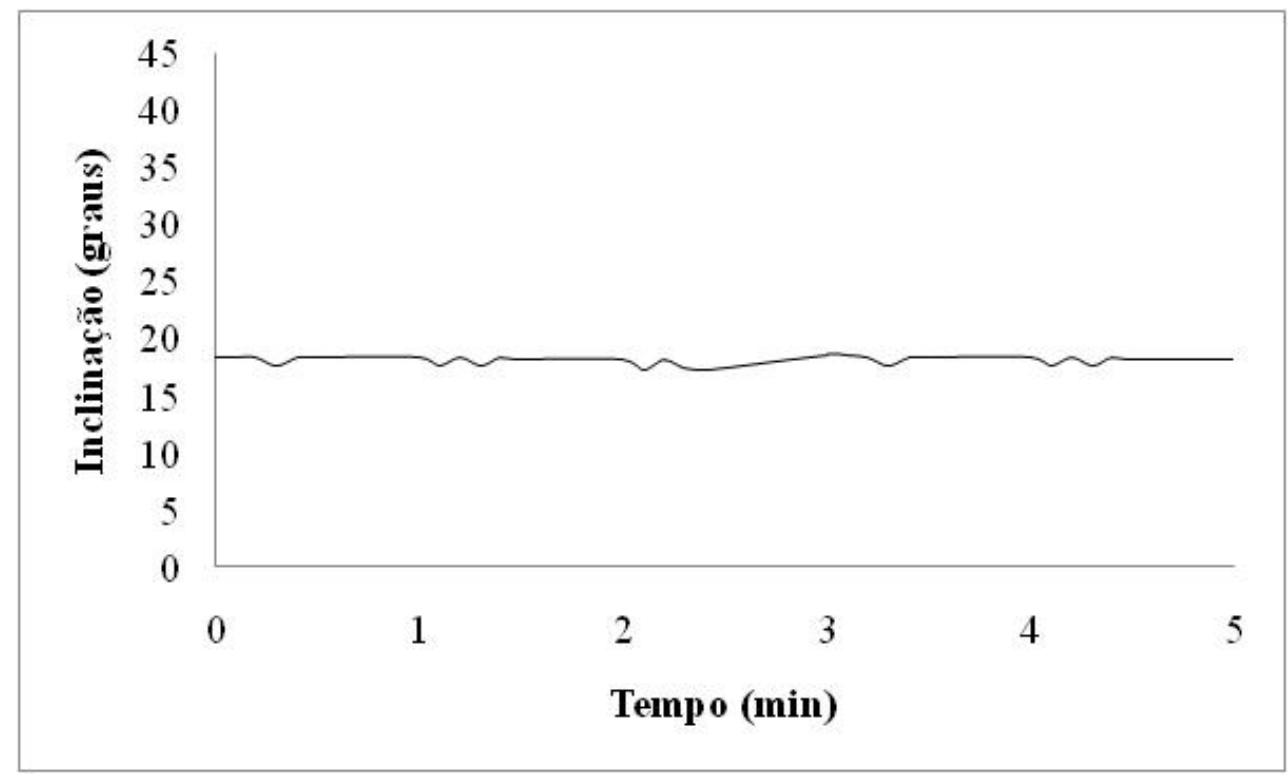

Figura 9 - Curva de estabilidade do inclinômetro para um intervalo de 5 minutos.

\section{CONCLUSÕES}

O inclinômetro construído e avaliado mostrou-se ser eficiente para indicação da inclinação do terreno em operações de campo com tratores agrícolas, apresentando pequena oscilação do ângulo, tempo de resposta suficiente e facilidade de visualização, auxiliando o operador na tomada de decisão para evitar um tombamento lateral da máquina nos diferentes terrenos em que o mesmo está operando.

\section{REFERÊNCIAS}

BENZ, D. et al. On low cost inclination sensors made from selectively metallized polymer. Sensors and Actuators, New York, A 123-124, p. 18-22, 2005.

BILLAT, S. et al. Micromachined inclinometer with high sensitivity and very good stability. Sensors and Actuators, New York, A 97-98, p. 125-130, 2002. 
CELESTE, W. C. Montagem e teste de inclinômetro microprocessados utilizando acelerômetros baseados em tecnologia MEMS. 70 p. Dissertação (Mestrado em Engenharia Elétrica) - Universidade Federal do Espírito Santo, Vitória, 2002.

CORRÊA, I. M.; RAMOS, H. H. Acidentes rurais. Cultivar Máquinas. Pelotas, n 16. p. 24-25, 2003.

CUTULI, J. A.; CAMPANUCCI, L.; TUSIANI, M. O. Seguridad y higiene en el trabajo. Buenos Aires: Instituto Argentino, 1977. 512 p.

GAIOTTO, J.R.C. Terreno inclinado. Cultivar Máquinas. Pelotas, n 24. p. 22-23. 2003.

HUNTER, A. A review of research into machine stability on slopes. [ S.1. ]: Agricultural- Engineer, 1992. n. 47 , p. $49-53$

MÁRQUEZ, L. Ergonomia y seguridad em los tractores agrícolas. In:_. Solo tractor’90. Madrid: Laboreo, 1990. cap. 4, p. 146-207.

MIALHE, L. G. Máquinas motoras na agricultura. São Paulo: EPU/USP, 1980, v.2, 290 p.

OT, F., LLADÓ, S. Inclinómetro económico (galgas extensiométricas). Sensors and Actuators, New York, n. 76, p. 139-146. 2001.

PINHO, M.S. Tópicos em computação gráfica: rastreamento. Porto Alegre: PUC, Faculdade de Ciências e Informática, 2005. Disponível em: <http://www.facin.pucrs.br/inclinometros.pucrs.htm>. Acesso em: $07 / \mathrm{mar} / 2005$. 
SANTOS, J. E. G. Curso de especialização em engenharia de segurança do trabalho, Módulo: Segurança Agropecuária. Bauru, Universidade Estadual Paulista, Faculdade de Engenharia Mecânica. 2004, 134 p. (Apostila).

SCHLOSSER, J. F., DEBIASI, H. Acidentes com tratores agrícolas: caracterização e prevenção. Santa Maria: UFSM, 2001. 86 p. (Caderno didático, n. 8).

SILVA, N. C. Pêndulo simples: modelo matemático. Baú de Ciências. UFSC, Departamento de Física. Disponível em: $<$ http:// www.fsc.ufsc.br/ canzian/bau/pendulo/pendulo-real.html $>$. Acesso em $13 / \mathrm{jul} / 2006$.

ZÓCCHIO, A. Prática da prevenção de acidentes: ABC da segurança do trabalho. 2. ed. São Paulo: Atlas, $1971.173 \mathrm{p}$. 